\title{
Avaliação do Descarte de Resíduos Sólidos no Meio Urbano. Estudo de Caso: Zona ZOEMI-APOC de Araraquara SP
}
Evaluation of Solid Waste Disposal in Urban Area. Case study: ZOEMI-APOC Area of Araraquara/SP

Avaluación de lo Desecho de Residuos Sólidos en la Area Urbana. Estudio de Caso: Zona ZOEMI-APOC de Araraquara/SP

Laura de Oliveira Battistini Pestana Graduanda de Engenharia Civil, UNESP Bauru, Brasil laura.pestana2201@gmail.com

Katia Sakihama Ventura

Professora DECiv - PPGEU, UFSCar, Brasil katiaventura@yahoo.com 


\section{RESUMO}

O descarte irregular de resíduos sólidos no meio urbano ocasiona diversos impactos ao meio, à saúde humana e à economia local. O objetivo geral deste artigo foi estruturar e aplicar método para mensuração de impactos ambientais, provenientes do depósito clandestino de resíduos sólidos. De acordo com o plano diretor urbanístico, a área de estudo está localizada na zona ZOEMI-APOC, pertencente à área urbana de Araraquara. O método proposto consistiu na elaboração e aplicação de três ferramentas (ficha de caracterização, lista de verificação e matriz GUT) para mensuração destes impactos em quatro pontos. Entre os resultados, os resíduos da construção civil se destacaram no estudo, especialmente no ponto 1, cujo volume representou $34 \%$ em relação ao total contabilizado. A maioria dos impactos da lista de verificação foi identificada nos pontos. A subjetividade do método pode ser minorada com a participação de especialistas e validação em outras áreas, em novas pesquisas. No entanto, isto não inviabilizou o uso da ferramenta para nortear o gestor público quanto aos desafios do tema. Ações como parceria com entidades de extensão e pesquisa, campanhas socioeducativas, planejamento a médio e longo prazos e uso de indicadores de serviços de limpeza urbana podem subsidiar a tomada de decisão para efetiva gestão de resíduos sólidos.

PALAVRAS-CHAVE: Resíduos Sólidos. Impactos Ambientais. Indicadores.

\section{ABSTRACT}

Improper disposal of solid waste in the urban area causes several impacts on the environment, human health and the local economy. The general objective of this paper was to structure and apply a method for measuring environmental impacts from solid waste deposit irregularly. According to urban planning, the site of study is located in ZOEMI-APOC, urban area of Araraquara. The proposed method consisted of the elaboration and application of three tools (characterization sheet, checklist and GUT matrix) to measure these impacts in four points. Among the results, construction waste stood out in the study, especially in point 1, whose volume represented $34 \%$ of the total accounted for. The most impacts of checklist were identified in the points. In new researches, expert's participation and validation in other areas for can minimized the subjectivity. However, the use of the tool to guide the public manager regarding the challenges of the theme was feasible. Actions such as partnership with extension and research entities, socioeducational campaigns, medium and long term planning and the use of urban cleaning services indicators can support decision making for effective solid waste management.

KEYWORDS: Solid waste. Environmental impacts. Indicators.

\section{RESUMEN}

La eliminación irregular de desechos sólidos en el area urbana causa varios impactos en el medio ambiente, la salud humana y la economía local. El objetivo general de este documento fue estructurar y aplicar un método para medir los impactos ambientales del depósito clandestino de residuos sólidos. El estudio fue localizado en la zona ZOEMI$A P O C$, perteneciente al área urbana de Araraquara. El método propuesto consistió en la elaboración y aplicación de tres herramientas (hoja de caracterización, lista de verificación y matriz GUT) para medir estos impactos in cuatro puntos. Entre los resultados, los residuos de construcción destacaran en el estudio, especialmente en el punto 1, cuyo volumen representaba el $34 \%$ del total contabilizado. La mayoría de los impactos de la lista de verificación se identificaron en los puntos. La subjetividad del método se puede disminuir con la participación de expertos y la validación en otras áreas para futuras investigaciones. Sin embargo, esto no impidió el uso de la herramienta para guiar al administrador público con respecto a los desafíos del tema. Acciones como la asociación con entidades de extensión e investigación, campañas socioeducativas, planificación a medio y largo plazo y el uso de indicadores de servicios de limpieza urbana pueden apoyar la toma de decisiones para una gestión eficaz de los residuos sólidos.

PALABRAS-CLAVES: Residuos Sólidos. Impactos Ambientales. Indicadores. 


\title{
INTRODUÇÃO
}

Gestão e gerenciamento de resíduos sólidos

A Associação Brasileira de Normas Técnicas (ABNT) define resíduos sólidos como (ABNT, 2004):

\begin{abstract}
Resíduos nos estados sólido e semissólido, que resultam de atividades de origem industrial, doméstica, hospitalar, comercial, agrícola, de serviços e de varrição. Ficam incluídos os lodos provenientes de sistemas de tratamento de água, aqueles gerados em equipamentos e instalações de controle de poluição, bem como determinados líquidos cujas particularidades tornem inviável o seu lançamento na rede pública de esgotos ou corpos de água, ou exijam para isso soluções técnica e economicamente inviáveis em face à melhor tecnologia disponível.
\end{abstract}

A Política Nacional de Resíduos Sólidos, Lei Federal n 12.305/2010, em seu artigo 13, classifica os resíduos sólidos quanto à origem e quanto à periculosidade, destacando, entre outros, algumas classes (BRASIL, 2010):

- Resíduos Domiciliares (RD): são aqueles originários de atividades realizadas em domicílios na área urbana;

- Resíduos de Limpeza Urbana (RLU): são resultantes da limpeza de área públicas, como varrição de feiras, poda de parques e praças, limpeza de vias públicas;

- Resíduos Sólidos Urbanos (RSU): englobam os domiciliares e de limpeza urbana;

- Resíduos da Construção Civil (RCC): aqueles gerados em construções, reformas, demolições de obras e edificações em geral;

Quanto à periculosidade, os resíduos sólidos podem ser considerados perigosos (Classe I) ou não perigosos (Classe II). Os de classe I apresentam uma ou mais propriedades que implicam em significativo risco à saúde pública ou à qualidade ambiental como toxicidade, reatividade, inflamabilidade, corrosividade e patogenicidade. Os resíduos Classe II são enquadrados em inertes e não inertes e ambas não possuem as características da classe anterior (ABNT, 2004). A mesma Lei, em seu artigo 3-, define o gerenciamento de resíduos sólidos, como as etapas de coleta, transporte, transbordo, tratamento, destino final adequado dos resíduos sólidos e disposição final apropriada dos rejeitos. Além disto, este item da Lei esclarece que o gerenciamento destes materiais deve estar apresentado no plano municipal de gestão integrada de resíduos sólidos, cujo conceito implica nas ações voltadas para iniciativas relacionadas às dimensões da sustentabilidade.

A responsabilidade pelo gerenciamento de cada tipo de resíduo é apresentada no Quadro 1. 
Quadro 1: Responsabilidade pelo gerenciamento de algumas classes de resíduo sólidos

\begin{tabular}{|l|c|}
\hline \multicolumn{1}{|c|}{ Tipo de Resíduos Sólidos } & Responsável \\
\hline Domiciliar & Prefeitura / autarquia municipal \\
\hline Comercial & Prefeitura / autarquia municipal \\
\hline Serviços públicos & Prefeitura / autarquia municipal \\
\hline Industrial & Gerador \\
\hline Serviços de Saúde & Gerador \\
\hline Serviços de Transporte & Gerador \\
\hline Agrossilvopastoril & Gerador \\
\hline Resíduos de Construção Civil & Gerador \\
\hline Radioativo & Gerador com base na CNEN* \\
\hline *Comissão Nacional de Energia Nuclear \\
Fonte: adaptado de JARDIM et. al, 1995.
\end{tabular}

Impactos ambientais dos resíduos sólidos no meio e na paisagem urbana

Segundo o artigo 10 da Resolução $n^{\circ}$ 001/86 do Conselho Nacional do Meio Ambiente (CONAMA), impacto ambiental pode ser entendido como (CONAMA, 1986):

Qualquer alteração das propriedades físicas, químicas, biológicas do meio ambiente, causada por qualquer forma de matéria ou energia resultante das atividades humanas que afetem diretamente ou indiretamente: a segurança, a saúde e o bem estar da população; as atividades sociais e econômicas; a biota; as condições estéticas e sanitárias ambientais e a qualidade dos recursos ambientais.

Com o desenvolvimento econômico, a ocupação das áreas urbanas e zonas de risco, o crescimento populacional e a revolução tecnológica, os impactos ambientais negativos têm se tornado crescentes. Boa parte é oriunda de costumes e hábitos da população devido ao padrão de vida, ao uso diversificado dos recursos hídricos e à elevada geração de resíduos sólidos no meio urbano. Estes fatores associados ao manejo inadequado ou insuficiente podem levar a cenários agravantes que modificam a paisagem natural e comprometem os ecossistemas (MUCELIN e BELLINI, 2008; GOUVEIA, 2012).

O país é habitado por cerca de 210 milhões de brasileiros (IBGE, 2019) e são coletados cerca 1,0 kg de resíduo domiciliar e de limpeza pública por habitante diariamente (MDR, 2019). Esse grande volume de RSU torna-se um problema devido à poluição visual e pode contribuir para desvalorização do imóvel, ameaçando os seres humanos e o meio ambiente, além de reduzir consideravelmente os espaços úteis disponíveis (SCHALCH et. al, 2002).

Os rejeitos podem ser descartados em aterros sanitários e os resíduos sólidos podem ser encaminhados a algum tipo de aproveitamento energético por meio de processamentos físicos e/ou químicos para a fabricação de novos produtos (MOTA et. al, 2009; BRASIL, 2012). 0 descarte incorreto de resíduos sólidos é realizado em vazadouros a céu aberto (popularmente conhecidos como "lixões"), mas podem ser submetidos à compostagem (fração orgânica), incineração (perigosos) ou reciclagem (potencial econômico ou energético), segundo Gouveia (2012). 
Nos resíduos sólidos descartados, estão presentes agentes físicos, químicos e biológicos capazes de causar interferências à saúde humana e ao meio ambiente (FERREIRA e ANJOS, 2001). Os mesmos autores esclarecem que, dentre os agentes físicos, o odor emanado pela decomposição orgânica associada ao grande volume de resíduos sólidos depositados de forma equivocada e em áreas irregulares amplia tais impactos.

Os agentes químicos são os resíduos químicos encontrados em diversos produtos de consumo, tais como pilhas e baterias, pesticidas, tintas, cosméticos, remédios, dentre outros. As principais rotas de exposição a estes contaminantes são a dispersão do solo e do ar contaminado, a lixiviação e a percolação do chorume. Dentre os compostos, uma parcela considerável pode ser classificada como perigosa devido à presença de metais que desencadeiam efeito acumulativo no organismo humano e podem provocar doenças como saturnismo, distúrbios no sistema nervoso, câncer, anomalias congênitas, etc. Ainda, podem comprometer a qualidade da água, do solo e do ar (KUPCHELLA e HYLAND, 1993; FERREIRA e ANJOS, 2001; GOUVEIA, 2012).

Os agentes biológicos, por sua vez, são responsáveis pela transmissão direta ou indireta de doenças, sendo a última dada pelos vetores que encontram nos resíduos condições adequadas e favoráveis à sua sobrevivência e proliferação. Além da presença de microrganismos patogênicos nos resíduos domiciliares, como lenços de papel, fraldas descartáveis e papel higiênico, os resíduos de serviços de saúde apresentem elevadas concentrações destes e, quando descartados indiscriminadamente no meio, prejudicam a sociedade como um todo (FERREIRA e ANJOS, 2001).

Isto implica em conhecimento prévio da segregação adequada, seguido de acondicionamento e armazenamento apropriados dos resíduos sólidos por tipo para que a população não se contamine ou amplie os impactos de modo geral. Cabe esclarecer que mecanismos de controle e monitoramento podem ser implementados pelo poder público ou por iniciativa privada em parceria com instituições de ensino para promover melhorias das condições ambientais e sanitárias nas cidades.

\section{Ferramentas de avaliação de impactos ambientais}

A avaliação de impacto ambiental (AIA) é um conjunto de estudos realizados a fim de "identificar, prever, interpretar e prevenir os efeitos ambientais que determinadas ações, planos, programas ou projetos podem causar à saúde, ao bem-estar humano e ao ambiente", segundo BOLEA (1984). É uma ferramenta de apoio à tomada de decisão que promove o conhecimento preliminar e a análise imparcial dos impactos positivos e negativos, de forma preventiva e voltada à otimização de benefícios (PIMENTEL et. al, 1992).

Existem algumas metodologias empregadas com esta finalidade e que se baseiam em um conjunto de diretrizes. Porém, a maioria dos métodos de avaliação de impactos ambientais apresentam caráter subjetivo na abordagem do meio físico. Para minorar tal limitação, devem ser utilizados critérios bem definidos para a escolha das notas atribuídas definindo uma escala de abrangência do assunto (CREMONEZ et. al, 2014).

Não é recomendável a escolha de um determinado método como o mais vantajoso para a avaliação dos impactos ambientais, uma vez que cada um apresenta suas características específicas, como vantagens e desvantagens, não existindo uma metodologia ideal que se aplique a todas as fases de análise. Para sua seleção, devem-se levar em conta diversos aspectos 
como a disponibilidade de dados, a localização e suas especificidades, tempo e recursos financeiros disponíveis (PIMENTEL et.al, 1992).

Entre os métodos, há modelos que consistem na simulação computacional com o uso de inteligência artificial ou modelos matemáticos para reproduzir tanto quanto possível o comportamento de parâmetros ambientais ou as inter-relações entre as causas e os efeitos de determinadas ações. São métodos úteis para projeto de usos múltiplos. Quanto à complexidade, depende da disponibilidade e qualidade dos dados e requer especialistas para o desenvolvimento de modelos matemáticos, pois há limitação de variáveis. Existem alguns pontos que necessitam de avaliação para evitar a tomada de decisão imperfeita (PIMENTEL et. al, 1992; CREMONEZ et. al, 2014).

Cabe observar que existem as metodologias quantitativas, as quais associam números e valores para explicar o comportamento de uma observação avaliada qualitativamente. Segundo Sabino (1996), a análise quantitativa resulta da informação numérica da investigação, baseado em um conjunto de quadros, tabelas e medidas. O método quantitativo pode utilizar indicadores (parâmetros) de qualidade ambiental expressos por gráficos, os quais relacionam os segmentos ambientais em estudo com seu respectivo estado de qualidade, a partir dos pesos atribuídos (FINUCCI, 2010; CREMONEZ et. al, 2014).

Os principais métodos encontram-se, resumidamente, apresentados no Quadro 2.

Quadro 2: Breve descrição de métodos de avaliação de impactos ambientais

\begin{tabular}{|c|l|}
\hline Método & \multicolumn{1}{c|}{ Descrição do Método } \\
\hline AD HOC & $\begin{array}{l}\text { Tomada de decisões para implantação de projetos, com base no parecer de especialistas em cada } \\
\text { espécie de impacto resultante do projeto, além dos pontos econômicos e técnicos. }\end{array}$ \\
\hline $\begin{array}{c}\text { Check-in } \\
\text { (Listagem) }\end{array}$ & $\begin{array}{l}\text { São listas padronizadas dos fatores ambientais para identificar os impactos possíveis. Algumas } \\
\text { incluem informações sobre técnicas de previsão de impacto, outras incluem descrição dos impactos } \\
\text { ou incorporam escalas de valor e índices de ponderação dos fatores. }\end{array}$ \\
\hline $\begin{array}{c}\text { Matrizes de } \\
\text { Interação }\end{array}$ & $\begin{array}{l}\text { Listagem de controle bidimensional que relaciona os fatores com as ações, sendo eficiente na } \\
\text { identificação de impactos diretos, uma vez que tem por objetivo relacionar as interações entre os } \\
\text { fatores ambientais e os componentes do projeto. }\end{array}$ \\
\hline Redes de & $\begin{array}{l}\text { Visa o estabelecimento de uma sequência de impactos ambientais provenientes de determinada } \\
\text { intervenção, representando-os utilizando gráficos. Podem ser utilizadas para orientar as medidas a } \\
\text { serem propostas para a minimização dos impactos observados. }\end{array}$ \\
\hline Superposição & $\begin{array}{l}\text { Métodos cartográficos para planejamento territorial. Cada mapa indica uma característica cultural, } \\
\text { social e física que refletem um impacto. Esses mapas quando integrados produzem a síntese da } \\
\text { situação ambiental de uma área geográfica. }\end{array}$ \\
\hline
\end{tabular}

Fonte: PIMENTEL et. al, 1992; CREMONEZ et. al, 2014.

Lakatos e Marconi (2011) apontam que as principais características do enfoque quantitativo:
a) evidencia a observação e a valorização dos fenômenos;
b) estabelece ideias;
c) demonstra o grau de fundamentação;
d) revista ideias resultantes da análise e,
e) propõe novas observações e valorizações para esclarecer, modifica e/ou fundamentar respostas e ideias. 
Estas autoras ainda reforçam a necessidade de observar a "objetividade, sistematização e quantificação dos conceitos, evidenciados na comunicação".

As vantagens e desvantagens das avaliações quantitativas apresentam-se no Quadro 3.

Quadro 3: Vantagens e desvantagens sobre métodos quantitativos de avaliação

\begin{tabular}{|c|c|}
\hline Vantagens & Desvantagens \\
\hline $\begin{array}{l}\text { Resultados fornecem boas informações para } \\
\text { caracterizar uma situação ambiental e prever } \\
\text { impactos. Permite envolvimento de diferentes } \\
\text { equipes de especialistas, diminuindo a } \\
\text { subjetividade. Explicita bases de cálculo dos índices } \\
\text { de julgamento de valor. Compara eficientemente } \\
\text { alternativas de um mesmo projeto. Facilita o } \\
\text { entendimento pela rapidez de interpretação. }\end{array}$ & $\begin{array}{l}\text { Requer excessivo trabalho preparatório para } \\
\text { estabelecer curvas das funções para cada } \\
\text { indicador ambiental. Pode não considerar a } \\
\text { base socioeconômica, os quais podem ser } \\
\text { difíceis de avaliar. Necessita de opinião de } \\
\text { especialistas, quando o método ou escala não } \\
\text { está reconhecido em literatura, para minimizar } \\
\text { os erros de avaliação ou inconsistência de } \\
\text { dados. }\end{array}$ \\
\hline
\end{tabular}

Fonte: PIMENTEL et.al, 1992; CREMONEZ et. al, 2014;

Entre os métodos quantitativos, tem-se o método GUT que representa a análise de três critérios: gravidade, urgência e tendência. É uma ferramenta utilizada para definir quais são as atitudes prioritárias (ou problemas a serem minimizados) dentro de um leque de alternativas de ação a partir de uma ordenação de importância destas de acordo com a sua gravidade, urgência e tendência em casos de ausência ou demora para a tomada de atitude (MEIRELES, 2001; FERREIRA et. al, 2018).

Para utilizar a ferramenta e estruturar a matriz GUT, Ferreira et.al (2018) e Meirelles (2001) recomendam a listagem prévia de impactos que possam ser observados no local de avaliação para que, posteriormente, auxilie a atribuição de notas aos seguintes critérios:

$\checkmark$ Gravidade (G): refere-se à intensidade ou profundidade que o problema / situação observada pode causar;

$\checkmark$ Urgência (U): considera a escala temporal para avaliação das consequências;

$\checkmark$ Tendência (T): o desenvolvimento que terá o problema.

$\checkmark$ Produto (P): representa a multiplicação dos critérios a partir da escala adotada. $O$ valor obtido indica que quanto maior a nota do problema observado, pior será o impacto avaliado.

\section{OBJETIVO}

O principal objetivo deste artigo foi estruturar e aplicar método de mensuração dos impactos ambientais provenientes do descarte irregular de resíduos sólidos em área urbana do município de Araraquara.

Os objetivos específicos foram:

- Elaborar um conjunto de formulários para caracterização dos impactos ambientais;

- Mensurar os impactos analisados nos pontos definidos. 


\section{MATERIAIS E MÉTODOS}

O estudo iniciou a partir do levantamento bibliográfico sobre a gestão e o gerenciamento de resíduos sólidos, os impactos ambientais destes no meio e as ferramentas de avaliação, instrumentos leais e normativos para fundamentação teórica.

Em seguida, foram elaboradas as ferramentas de avaliação dos impactos para cada ponto:

- Ficha de caracterização das observações em campo: possibilitou o registro de fotografias, a identificação da coordenada geográfica do ponto, os tipos de resíduos sólidos descartado, a inserção de croqui para posterior análise quantitativa do volume estimado pelo descarte;

- Lista de verificação: identificação dos principais impactos que podem ser gerados pelo descarte de resíduos sólidos;

- Matriz GUT: método adotado para avaliação dos impactos observados, como apresentado no item anterior. A escala adotada se baseou no estudo de Oliveira (2017) que foi 1 (baixo), 2 (médio) e 3 (alto). Assim, quanto maior o valor obtido, mais atenção o local merece do poder público.

Cabe esclarecer que estes materiais estão apresentados no item "Resultados e Discussão" com os respectivos dados preenchidos. Além disto, a listagem de impactos ao meio, à saúde humana e à economia foi adaptada de Oliveira (2017) que aplicou seus instrumentos em 78 pontos, abrangendo cerca de $5 \mathrm{~km}^{2}$ em São Carlos, localizado a $30 \mathrm{~km}$ de Araraquara.

Cabe observar que de Araraquara, desde a instituição da Taxa de Resíduos Sólidos, Lei Municipal $\mathrm{n}^{\circ} 8556 / 2015$, recebe investimentos e melhorias no setor (Araraquara, 2015). Assim, foram definidos os seguintes critérios para seleção da área:

- Proximidade com a área urbana, podendo sofrer influência da zona rural pela aproximação com a cidade;

- Locais previamente conhecidos pelo acúmulo, mas em situação temporária ou contínua de depósito clandestino de materiais em questão;

- Fácil acesso e visualização que favoreça o registro de fotos, garantindo a segurança do grupo de pesquisa.

A interferência da paisagem urbana foi analisada com base nos impactos vistos em campo. Destaca-se que o grupo de pesquisa adotou parâmetros qualitativos associados à análise quantitativa pois, desta forma, viabiliza-se o controle das observações e os aspectos analisados são mais facilmente compreendidos pelo gestor.

A fim de caracterizar o objeto de estudo, foram empregados o Google Maps para servir de apoio à imagem aérea dos pontos visitados (Google Maps, 2019), o plano municipal de saneamento para compreensão da gestão de resíduos sólidos e os serviços públicos de limpeza urbana (ARARAQUARA, 2014) e o plano diretor urbanístico de Araraquara para identificação da área no zoneamento municipal (ARARAQUARA, 2014).

Por fim, foram propostas ações para auxiliar a gestão dos resíduos sólidos e manter a preservação socioambiental das áreas afetadas. 


\section{RESULTADOS E DISCUSSÃO \\ Objeto de Estudo}

Segundo dados levantados no Plano Municipal de Gestão Integrada de resíduos sólidos, Araraquara está localizado no interior do estado de São Paulo e situa-se na região Central do Estado a uma distância de $43 \mathrm{~km}$ do seu centro geográfico (Obelisco) e a 277 quilômetros da Capital (cidade de São Paulo). O município está conurbado com Américo Brasiliense (DAAE, 2013).

A cidade possui uma área total de $1.006 \mathrm{~km}^{2}$, sendo cerca de $77,37 \mathrm{~km}^{2}$ de área urbana. Destes, aproximadamente $39 \mathrm{~km}^{2}$ são relativos à área urbana consolidada (DAAE,2013) e possui cerca de 236.072 habitantes (IBGE CIDADES, 2019).

A cidade de Araraquara é dividia em 8 zonas principais, sendo elas (ARARAQUARA, 2014):

- Categoria Zonas Ambientais (ZAMB)

- ZOPA (Zonas de Proteção Ambiental)

- ZAUS (Zonas Ambientais de Uso Sustentável)

- ZORA (Zona de Conservação e Recuperação Ambiental)

- Categoria Zonas de Estruturação Urbana Sustentável (ZEUS)

- ZOPRE (Zonas Predominantemente Residenciais)

- ZOEMI (Zonas Especiais Mistas)

- ZEPP (Zonas Especiais Predominantemente Produtivas)

- CPE (Corredores de Produção Econômica)

- ZORUR (Área Urbana, predominantemente agrária)

As Figuras 2 e 3 permitem a visualização aérea do objeto de estudo, com os pontos definidos pelo grupo de pesquisa. O método foi aplicado no trecho em estudo, o qual foi organizado em 4 pontos (Figura 3 ).

A região de estudo está localizada na zona ZOEMI-ACOP (Zonas Especiais Mistas - Área da Cidade Compacta de Ocupação Prioritária) em destaque na Figura 1. Esta, segundo a Lei Complementar $\mathrm{n}^{\circ}$ 850, de 11 de fevereiro de 2014, é caracterizada por ser (ARARAQUARA, 2014):

[...] área da Cidade Compacta de Ocupação Prioritária, representando a maior subdivisão territorial do zoneamento e modelo espacial, e uma das zonas prioritárias e estratégicas de indução e consolidação do processo de urbanização, estimulando a edificabilidade do solo urbano através dos instrumentos do Estatuto da Cidade previstos nesta Lei, e ocupação de imóveis urbanos ociosos, não utilizados ou subutilizados, segundo conceitos da cidade sustentável. 
Figura 1: Recorte da área em estudo

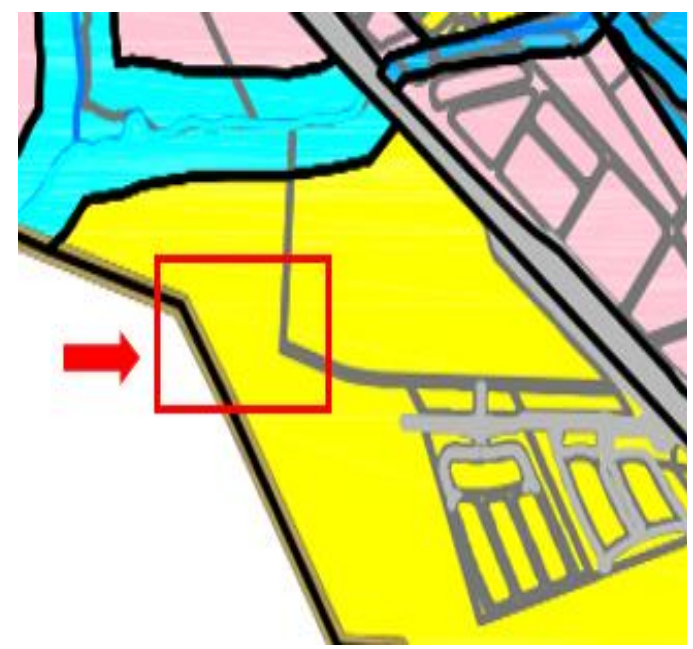

Fonte: ARARAQUARA, 2013
Figura 2. Vista aérea do objeto de estudo

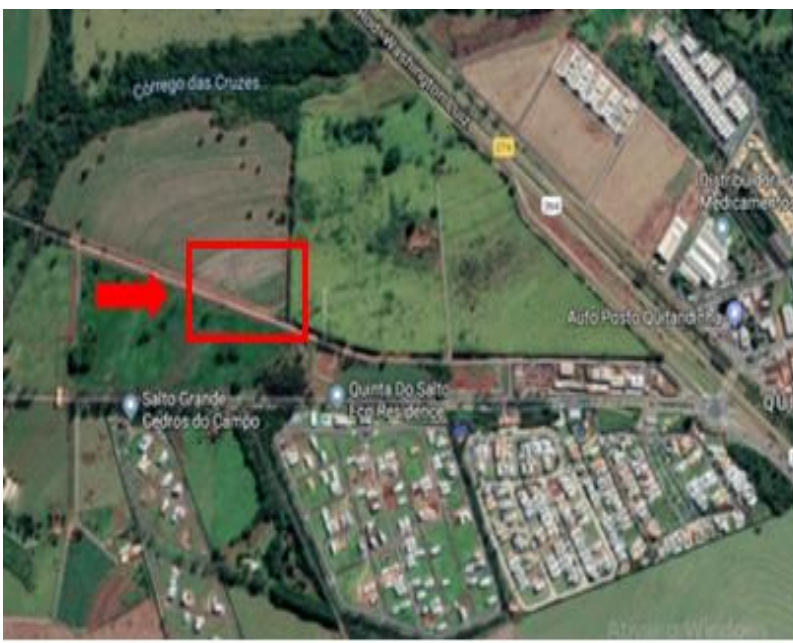

Fonte: Google Maps, 2019

Figura 3: Vista aérea com locação dos pontos analisados

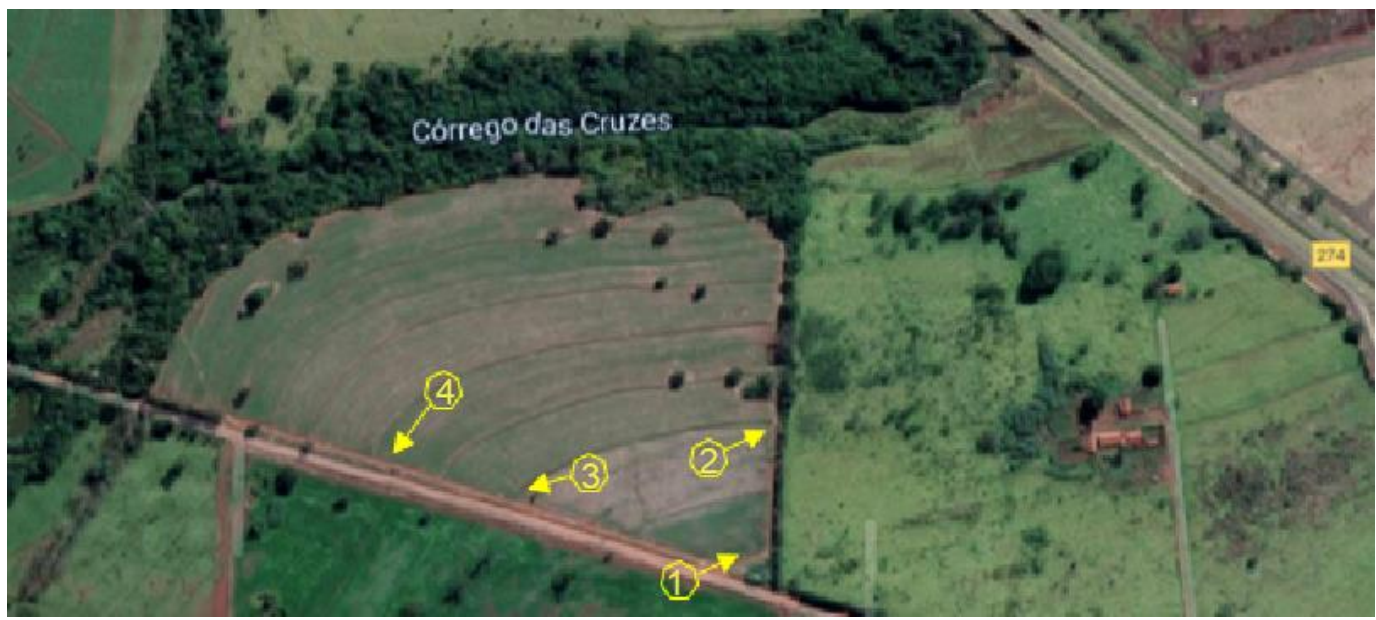

Fonte: pesquisadores, 2019.

A gestão de resíduos sólidos em Araraquara é de responsabilidade do Departamento Autônomo de Água e Esgotos (DAAE). Isto significa que a atuação da cooperativa de catadores, coleta seletiva, a operação do Aterro Sanitário (AS), a coleta e o transporte de RSU estão entre o rol de atribuições deste Departamento (ARARAQUARA, 2014).

A operação do AS de Araraquara está desativada pela finalização da obra e, portanto, transporta os RSU até o município de Guatapará, localizado a $54 \mathrm{~km}$, para disposição em aterro particular (ARARAQUARA, 2014). O município possui Taxa de Resíduos Sólidos (TRS) e, conforme o artigo 5o, está prevista a Categoria Residencial Social, destinada aos que estão adimplentes com o DAAE e incluídos no Cadastro Único para Programas Sociais - CadÚnico. Para estas situações, foram garantidos os descontos para à Categoria Residencial, da seguinte forma (ARARAQUARA, 2015):

- $40 \%$ para a faixa de produção de 0 a $20,1 \mathrm{~kg}$; 
- $0 \%$ para a faixa de produção de 20,2 a $40,2 \mathrm{Kg}$.

\section{Aplicação do Método para Avaliação da Paisagem Local}

Os dados registrados em campo encontram-se descritos nos Quadros 4 a 7, cuja estruturação da ficha de caracterização foi elaborada pelos presentes pesquisadores, como apresentado no item anterior.

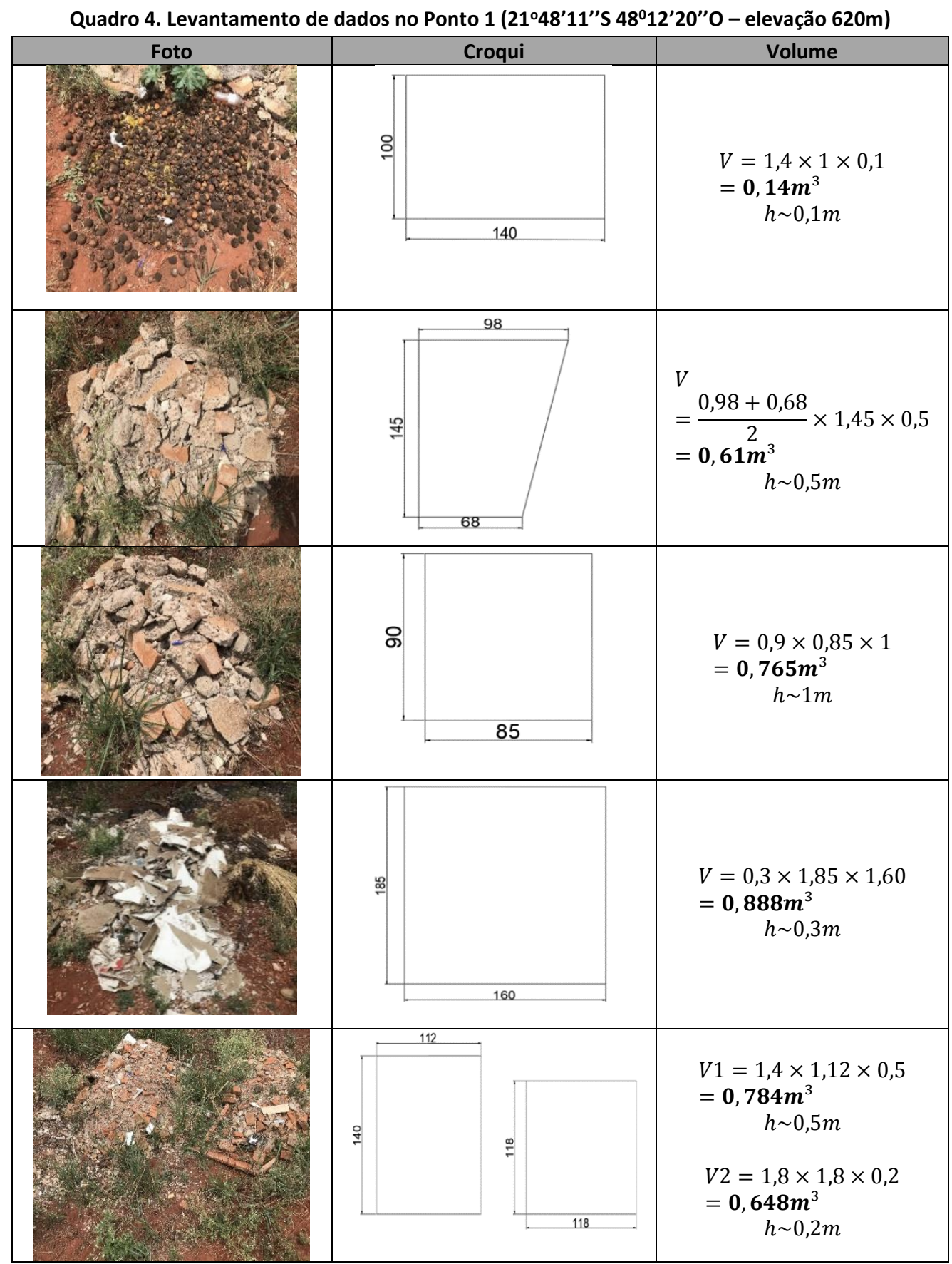




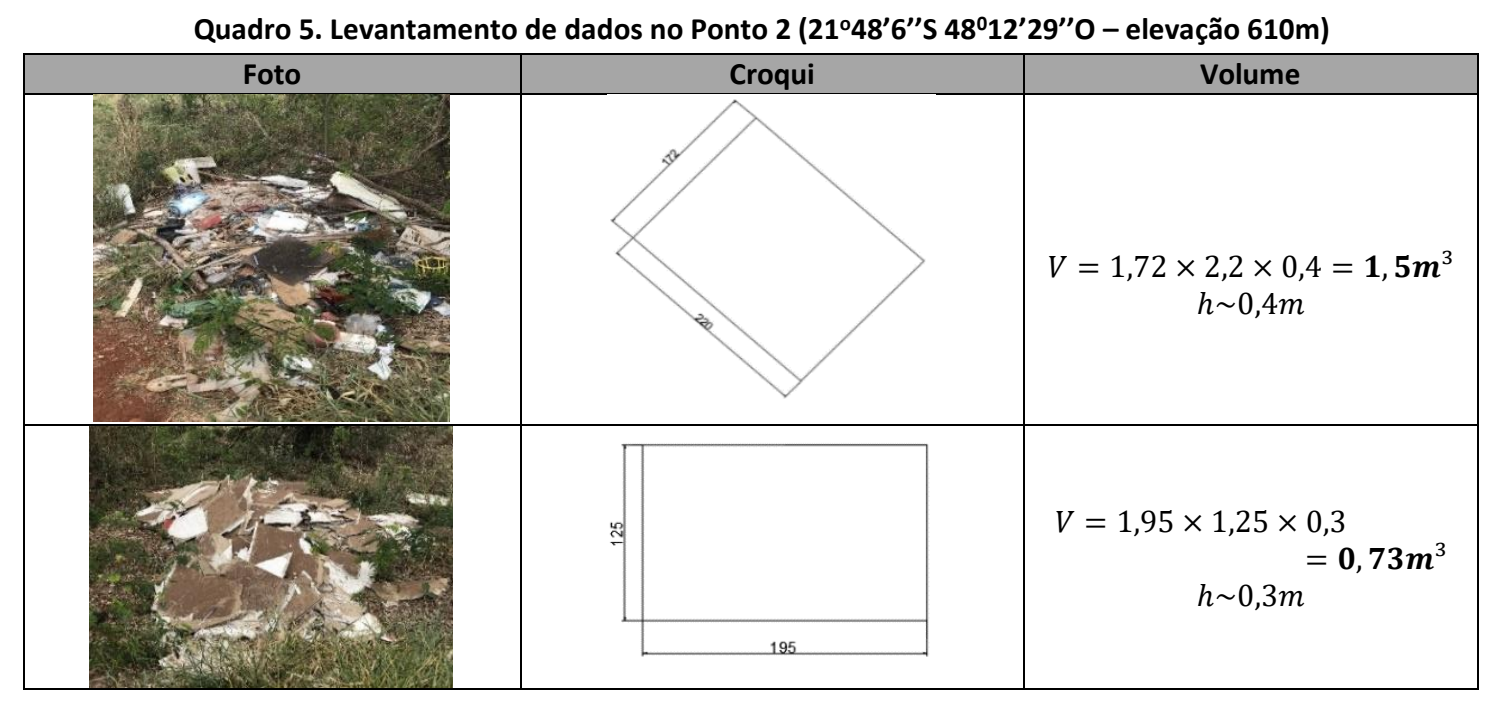

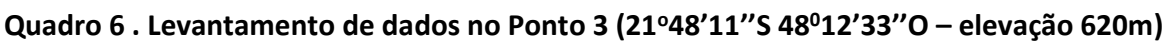

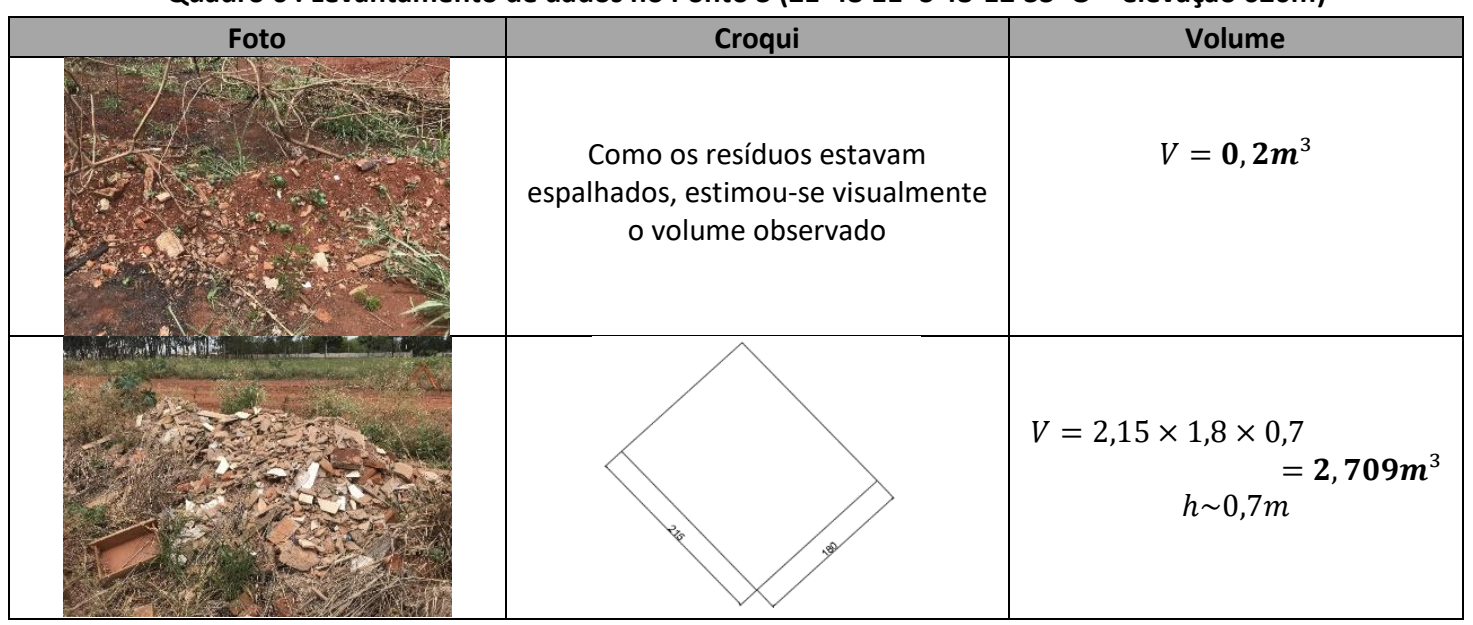

Quadro 7. Levantamento de dados no Ponto 4 (2148'11"'S 48012'34"O - elevação 610m)

\begin{tabular}{|c|c|}
\hline Foto & Colume \\
\hline & $V=\frac{3,2 \times 1,4}{2} \times 0,3$ \\
$=\mathbf{0 , 6 7 2 m ^ { 3 }}$ \\
$h \sim 0,3 m$ \\
\end{tabular}




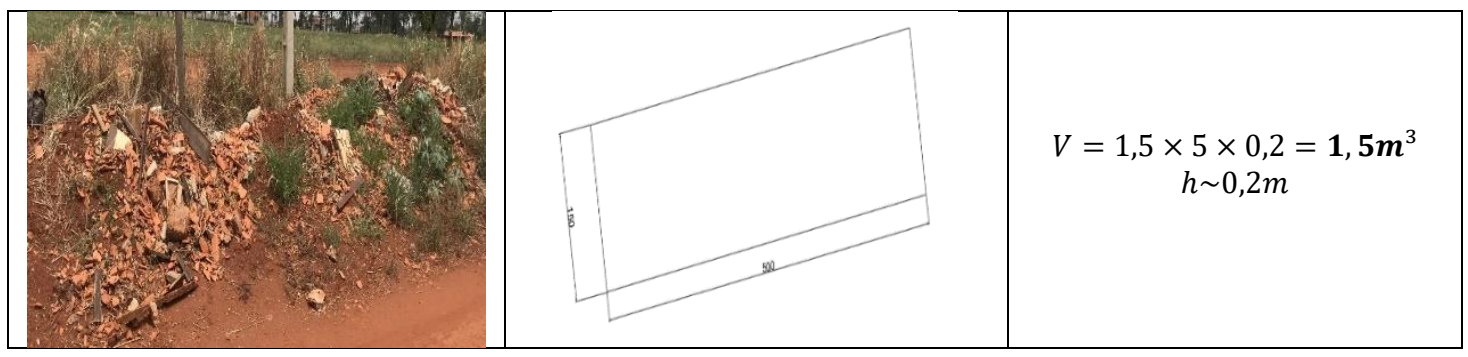

O Quadro 8 apresenta a lista de verificação dos impactos observados em visita a campo.

Quadro 8. Checklist dos impactos identificados nos pontos observados

\begin{tabular}{|c|c|c|c|c|c|c|c|c|c|}
\hline Impacto no meio & P1 & $\mathbf{P 2}$ & P3 & P4 & Impacto na economia & P1 & $\mathbf{P 2}$ & P3 & P4 \\
\hline Mau cheiro & $x$ & $x$ & $x$ & $\mathrm{x}$ & $\begin{array}{l}\text { Custo de manutenção com } \\
\text { limpeza }\end{array}$ & $\mathrm{X}$ & $\mathrm{x}$ & $\mathrm{x}$ & $\mathrm{x}$ \\
\hline Esgotamento dos recursos naturais & & & & & Desvalorização do imóvel & $x$ & $\mathrm{X}$ & $\mathrm{x}$ & $\mathrm{x}$ \\
\hline Proliferação de vetores & $\mathrm{x}$ & $x$ & $x$ & $x$ & Dificuldade para venda do & & & & \\
\hline Poluição do ar & $x$ & $x$ & $x$ & $x$ & imóvel & $x$ & $x$ & $x$ & $x$ \\
\hline Atração de outros tipos de resíduos & $x$ & $x$ & $\mathrm{x}$ & $x$ & $\begin{array}{l}\text { Redução do aproveitamento } \\
\text { de área útil do imóvel }\end{array}$ & $x$ & $\mathrm{X}$ & $\mathrm{x}$ & $x$ \\
\hline Proliferação de animais peçonhentos & $x$ & $\mathrm{X}$ & $x$ & $x$ & Impacto na saúde humana & P1 & P2 & P3 & P4 \\
\hline Prejuízo à paisagem & $x$ & $x$ & $x$ & $\mathrm{x}$ & $\begin{array}{l}\text { Obstrução de vias e } \\
\text { logradouros públicos }\end{array}$ & $\mathrm{X}$ & $\mathrm{X}$ & $x$ & $\mathrm{x}$ \\
\hline Poluição da água & & & & & Ameaça à saúde pública & $\mathrm{x}$ & $\mathrm{X}$ & $x$ & $\mathrm{X}$ \\
\hline Percolação de chorume & $x$ & $x$ & $x$ & $x$ & Prejuízo à qualidade de vida & $x$ & $x$ & $x$ & $x$ \\
\hline Depósito/Concentração de metais & & $\mathrm{X}$ & & & Sensação de insegurança & $x$ & $x$ & $\mathrm{X}$ & $\mathrm{x}$ \\
\hline $\begin{array}{c}\text { Dificuldade na implantação/realização } \\
\text { de coleta seletiva }\end{array}$ & $x$ & $\mathrm{x}$ & $x$ & $\mathrm{x}$ & \multirow{2}{*}{\multicolumn{5}{|c|}{$\begin{array}{l}\text { Observação Geral: } \\
\text { - A maioria dos impactos listados foi observada } \\
\text { em todos os pontos. } \\
\text { - O ponto } 1 \text { localiza-se mais próximo à rodovia, o } \\
\text { que pode favorecer o descarte e o maior } \\
\text { acúmulo e diversidade de materiais. }\end{array}$}} \\
\hline $\begin{array}{c}\text { Presença de substâncias tóxicas } \\
\text { (resinas, tintas) }\end{array}$ & & $x$ & & $x$ & & & & & \\
\hline
\end{tabular}

Os impactos ambientais caracterizados anteriormente e estimados encontram-se na matriz GUT (Tabela 1).

Tabela 1. Matriz GUT para os impactos observados no trecho analisado da Zona Mista de Araraquara / SP

\begin{tabular}{|c|c|c|c|c|c|c|c|c|c|c|c|c|c|c|c|c|}
\hline \multirow{3}{*}{$\begin{array}{l}\text { Impactos Ambientais observados em } \\
\text { campo }\end{array}$} & \multicolumn{16}{|c|}{ TRECHO } \\
\hline & \multicolumn{4}{|c|}{ Ponto 1} & \multicolumn{3}{|c|}{ Ponto 2} & & \multicolumn{4}{|c|}{ Ponto 3} & \multicolumn{4}{|c|}{ Ponto 4} \\
\hline & G & $\mathbf{U}$ & $\mathbf{T}$ & $\mathbf{R}$ & G & $\mathbf{U}$ & $\mathbf{T}$ & $\mathbf{R}$ & G & u & $T$ & $\mathbf{R}$ & G & $\mathbf{U}$ & $\mathbf{T}$ & $\mathbf{R}$ \\
\hline Presença de material orgânico & 1 & 1 & 1 & 1 & 1 & 1 & 1 & 1 & 1 & 1 & 1 & 1 & 1 & 1 & 1 & 1 \\
\hline Presença de RCC e queimada & 3 & 2 & 2 & 12 & 2 & 3 & 2 & 12 & 2 & 2 & 2 & 8 & 2 & 2 & 2 & 8 \\
\hline $\begin{array}{l}\text { Depósito de material orgânico, atração de } \\
\text { vetores e animais peçonhentos }\end{array}$ & 2 & 2 & 2 & 8 & 2 & 2 & 2 & 8 & 2 & 2 & 2 & 8 & 2 & 2 & 2 & 8 \\
\hline Descarte de RCC & 3 & 2 & 1 & 6 & 1 & 2 & 2 & 4 & 1 & 2 & 2 & 4 & 1 & 2 & 2 & 4 \\
\hline $\begin{array}{l}\text { Presença de material inservível (papel } \\
\text { higiênico) e perigosos }\end{array}$ & 1 & 1 & 2 & 2 & 2 & 1 & 2 & 4 & 2 & 1 & 2 & 4 & 1 & 1 & 2 & 2 \\
\hline
\end{tabular}

Quadro 9. Síntese dos dados analisados nos respectivos pontos 


\begin{tabular}{|c|c|c|c|}
\hline Ponto & Volume $\mathbf{( m}^{\mathbf{3}} \mathbf{)}$ & $\mathbf{\%}$ & Risco observado no ponto \\
\hline 4 & 2,172 & 19,4 & Presença de RCC e queimada; resíduos orgânicos e presença de vetores \\
\hline 3 & 2,971 & 26,5 & Presença de RCC e queimada; resíduos orgânicos e presença de vetores \\
\hline 2 & 2,210 & 19,9 & Descarte de RCC e queimada \\
\hline 1 & 3,835 & 34,2 & Presença de RCC e queimada; resíduos orgânicos e presença de vetores \\
\hline TOTAL & $\mathbf{1 1 , 2 0 8}$ & $\mathbf{1 0 0}$ & \\
\hline
\end{tabular}

Assim, observa-se que o trecho analisado foi avaliado pela ferramenta adaptada de Oliveira (2017) e apontou o ponto 1, do trecho em estudo, como aquele que - por estar mais próximo à Rodovia Washington Luiz - está mais suscetível ao descarte clandestino de materiais como os aqui estudados.

O ponto 1 destacou-se com a estimativa de $34,2 \%$ do volume de resíduos sólidos encontrados no trecho, que são oriundos de obras e reformas da construção civil. Neste sentido, propõe-se que o município invista em operações constante de limpeza de resíduos volumosos e, em especial, em locais distantes dos bolsões. Por exemplo, como o município possui a Taxa de Resíduos Sólidos, é possível:

i) Instituir mutirão de limpeza urbana com planejamento físico-financeiro e com parceria de outras entidades para garantir limpa as zonas mais esquecidas ou pouco atendidas com serviços desta natureza;

ii) Ampliar a capacidade dos bolsões (locais que recebem geração de pequenos geradores de resíduos sólidos, isto é, até $1 \mathrm{~m}^{3}$ por pessoa ao dia) e, especialmente,

iii) Incentivar e auxiliar os geradores à correta segregação de materiais, diretamente na fonte em que eles são gerados.

Cabe destacar que placas informativas podem ser implementadas como alerta ao gerador do descarte clandestino. Além disto, ações de pesquisa e extensão podem ser desenvolvidas para que a sociedade receba apoio apropriado e atenda às diretrizes preconizadas da Política Nacional de Resíduos Sólidos como o controle social, gestão compartilhada e acordos setoriais para que a logística reversa funcione bem e de forma articulada com todos os agentes envolvidos. O uso de indicadores sobre os serviços de limpeza urbana e a melhoria da paisagem urbana também representam meios de controle socioambiental.

Outro instrumento útil é o uso de mapeamento (por exemplo, Sistema de Informação Geográfica - SIG) para identificar os pontos, as alterações da paisagem e as medidas adotadas para solucionar tal situação. Por fim, é essencial que o poder público disponibilize dados e realize conferências municipais para que a sociedade se aproprie deste conhecimento e possa, assim, auxiliar a tomada de decisão no planejamento urbano-ambiental, a longo prazo.

\section{CONCLUSÕES}

A gestão de resíduos sólidos urbanos tem sido vista como um problema para a sociedade em geral, mas pode ser compreendida como uma oportunidade para sanar e, efetivamente, direcionar esforços ao cumprimento da Política Nacional de Resíduos Sólidos. 
O estudo atingiu o objetivo principal, pois o modelo proposto caracterizou e estimou as informações qualitativas e quantitativas estabelecidas neste documento. $O$ instrumento elaborado mostrou-se útil para nortear o gestor público.

As ações, minimamente, propostas podem conduzir à reflexão da problemática observada.

Mesmo com a experiência profissional da equipe de pesquisadores e o apoio da literatura científica, o principal ponto negativo refere-se à ligeira subjetividade na ponderação de valores do método, que podem ser minorados com o apoio de especialistas. Outros métodos estatísticos e matemáticos podem corroborar com o refinamento do método.

No ponto 1, 34\% do volume estimado irregularmente foi oriundo do descarte de RCC. Para isto, ressalta-se a necessidade de implementação de sistema de monitoramento em escala municipal, com apoio da população e demais agentes parceiros para que a paisagem urbana se mantenha preservada e motive a sociedade ao convívio das áreas livres.

\section{AGRADECIMENTO}

Aos departamentos de Engenharia Civil da Universidade Federal de São Carlos (UFSCar) e Universidade Estadual Paulista (UNESP-Bauru).

\section{REFERÊNCIAS BIBLIOGRÁFICAS}

ARARAQUARA. LEI MUNICIPAL No 8.556, DE 09 DE OUTUBRO DE 2015. Fixa o valor da Taxa de Resíduos Sólidos. Araraquara: 2015.

ARARAQUARA. Plano municipal de saneamento básico. Araraquara: 2014. Disponível em <http://www3.araraquara.sp.gov.br/ImageBank/FCKEditor/file/administrador/1a\%20PMSB_Consolidado.pdf>. Acesso em:23 out. 2019.

ABNT - ASSOCIAÇÃO BRASILEIRA DE NORMAS TÉCNICAS. NBR 10004: Resíduos sólidos - classificação. Rio de Janeiro, p. 1. 2004.

ARARAQUARA. LEI COMPLEMENTAR № 850, DE 11 DE FEVEREIRO DE 2014. Da política de desenvolvimento municipal. Araraquara, $\quad$ SP, fev $2014 . \quad$ Disponível $\quad$ em <http://www3.araraquara.sp.gov.br/Pagina/Default.aspx?IDPagina=3973>. Acesso em: 18 out. 2019.

BRASIL. LEI № 12305, DE 2 DE AGOSTO DE 2010. Institui a política nacional de resíduos sólidos. Brasília, DF, ago 2010. Disponível em: <http://www.planalto.gov.br/ccivil_03/_ato2007-2010/2010/lei/l12305.htm>. Acesso em: 16 out. 2019.

CONAMA - Conselho Nacional de Meio Ambiente - Resolução n001, de 23 de janeiro de 1986. Publicado no D.O.U. de 17 de fevereiro de 1986.

BRASIL. Plano Nacional de Resíduos Sólidos. Brasília: MMA, 2012. Disponível em: <https://sinir.gov.br/images/sinir/Arquivos_diversos_do_portal/PNRS_Revisao_Decreto_280812.pdf>

BOLEA, T. Evaluación del impacto ambiental. Madrid, Mapfre, 1984.

CREMONEZ, Filipe Eliazar; CREMONEZ, Paulo André; FEROLDI, Michael; DE CAMARGO, Mariele Pasuch.; KLAJN, Felipe Fernandes; FEIDEN, Armin. Avaliação de impacto ambiental: metodologias aplicadas no Brasil. Revista Monografias Ambientais - REMOA v.13, n.5, dez, p.3821-3830. 2014.

FERREIRA, João Alberto; DOS ANJOS, Luiz Antonio. Aspectos de saúde coletiva e ocupacional associados à gestão dos resíduos sólidos municipais. Cad. Saúde Pública, Rio de Janeiro, 17(3):689-696, mai-jun, 2001. 
FERREIRA, Rafael Henrique Mainardes; BONET, Juliane; SILVA, Graziella dos Santos Portes. Diagnóstico Ambiental e Priorização de Impactos na Utilização da Matriz GUT: Análise em Indústria de Carvão Ativado de Guarapuava-PR. VII Congresso Brasileiro de Engenharia de Produção, dez. 2018.

FINUCCI, Marcelo. Metodologias utilizadas na avaliação do impacto ambiental para a liberação comercial do plantio de transgênicos. 230f. Dissertação (Mestrado), Programa de Pós-graduação em Saúde Pública, Faculdade de Saúde Pública da Universidade de São Paulo. São Paulo-SP, 2010.

GOUVEIA, Nelson. Resíduos sólidos urbanos: impactos socioambientais e perspectiva de manejo sustentável com inclusão social. 2012.

IBGE. Pesquisa Nacional de Saneamento Básico, PNSB -2008. Rio de Janeiro: IBGE;2010

IBGE. Site IBGE - População estimada. Disponível em: https://ibge.gov.br/. 2019.

IBGE Cidades. Panorama Araraquara. Disponível em: <https://cidades.ibge.gov.br/brasil/sp/araraquara/panorama > Acesso em 20 out. 2019.

JARDIM, Niza Silva et al. Lixo Municipal: manual de gerenciamento integrado. São Paulo: Instituto de Pesquisas Tecnológicas (IPT), e Compromisso Empresarial para Reciclagem (CEMPRE), 278p. 1995.

KUPCHELLA, Charles; HYLAND, Margaret. Environmental Science - Living Within the System of Nature. London: Prentice-Hall International. 1993.

LAKATOS, Eva Maria; MARCONI, Marina de Andrade. Metodologia científica. Editora Atlas: 6a ed. rev. e ampliada, 314p. 2011.

MEIRELES, Manuel. Ferramentas administrativas para identificar, observar e analisar problemas. 1. ed. São Paulo: Arte \& Ciência, 2001. Bibliografia: p. 51-58.

MOTA, José Carlos; DE ALMEIDA, Mércia Melo; DE ALENCAR, Vladimir Costa; CURI, Wilson Fadlo. Características e impactos ambientais causados pelos resíduos sólidos: uma visão conceitual. I Congresso Internacional de Meio Ambiente Subterrâneo. 2009.

MDR. SNIS Resíduos Sólidos 2019 - ano base 2017. Disponível em: <www.snis.gov.br>.

MUCELIN, Carlos Alberto; BELLINI, Marta. Lixo e impactos ambientais perceptíveis no ecossistema urbano. Sociedade \& Natureza, Uberlândia, 20(1): 111-124, jun. 2008.

OLIVEIRA, Tales Cristiano. Avaliação de impactos ambientais pelo descarte clandestino de resíduos sólidos na região norte em São Carlos - SP. São Carlos: Universidade Federal de São Carlos, 2017.

DAAE. Plano Municipal de Saneamento Básico - Plano Municipal de Gestão Integrada de Resíduos Sólidos. Araraquara, 2013

PIMENTEL, G.; PIRES, S.H. Metodologias de avaliação de impacto ambiental: aplicações e seus limites. Rev.Adm.púb, Rio de Janeiro, 26 (1): 56-68, jan./mar.1992.

SABINO, Carlos. El proceso de investigación. Buenos Aires: Lumen-Humanitas, 1996.

SCHALCH, Valdir; LEITE, Wellington Cyro de Almeida; JÚNIOR, José Leomar Fernandes; DE CASTRO, Marcus Cesar Avezum Alves. Gestão e gerenciamento de resíduos sólidos. São Carlos, out. 2002. 
